\title{
Modeling and Simulation of the Short-term Arterial Pressure Control System using an Object Oriented Approach
}

\author{
J. Fernandez de Canete, V. Muñoz-Martinez, J. Luque, J. Barbancho and J. Rozan
}

\begin{abstract}
Object-oriented modeling is spreading in current simulation environments through the use of the individual components of the model and its interconnections to define the underlying dynamic equations. In this paper we describe the use of the SIMSCAPE simulation environment in the objectoriented modeling of the short-term arterial pressure control system when is applied to patients undergoing different situations as hemorrhage, liquid uptake or hemodialysis treatment. Results show the effectiveness of the baroreceptor control mechanism to compensate for the hypotension induced by the hemodialysis treatment. The described approach represents a valuable tool in the teaching of physiology for graduate medical and biomedical engineering students when facing to alternate hemodialysis treatment.
\end{abstract}

Keywords- Cardiovascular Control, Baroreceptor system, Object-oriented Modeling, SIMSCAPE

\section{INTRODUCTION}

The mechanisms responsible for maintaining arterial blood pressure may be divided into short-term processes, which are effective over a period of seconds to hours, and mid- and long-term processes, which operate over days to weeks [1].The short-term mechanisms are based on neural control through the receptors in the heart and blood vessels, which sense blood pressure, together with the autonomic nervous system (ANS), which regulates cardiac function and the diameter of the resistance and capacitance vessels. The mid-term control is basically of a hormonal nature whereas the renal system would play the central role in long-term control in case of healthy subjects.

J. Fernandez de Canete, $\mathrm{PhD}$. is with the System Engineering and Automation Dpt., Engineering School, University of Malaga, 29071 Malaga, SPAIN (Phone 34-951329 ; Fax: 34-952133336; e-mail: ffernandezr@uma.es).

$\mathrm{V}$. Muñoz-Martinez $\mathrm{PhD}$. is with the System Engineering and Automation Dpt., Engineering School, University of Malaga, 29071 Malaga, SPAIN (e-mail:vfmm@ uma.es).

J. Luque PhD. is with the Dpt. Electronics, Computer Science School, University of Seville, 41010 Seville, SPAIN (e-mail: jluque@us.es).

J. Barbancho PhD. is with the Dpt. Electronics, Computer Science School, University of Seville, 41010 Seville, SPAIN (e-mail: jbarbancho@us.es).

J. Rozan collaborates with the System Engineering and Automation Dpt., Engineering School, University of Malaga, 29071 Malaga, SPAIN (email: johnrozan@alu.uma.es).
Cardiovascular modelling and control present a particular challenge and require both a multi-scale and multi-physics approach [2]. Several mathematical models of the closed loop cardiovascular system have been developed [3-4] and distinct simulation development tools have been used for graphic notation of the structure of physiological regulation systems, including that of the cardiovascular control system [5-7].

Short-term control of arterial pressure is achieved by the baroreceptor system which regulates the heart rate, the ventricular elastances, the cava elastance and the peripheral system resistance as a function of the mean aortic pressure value. When the cardiovascular system is stressed by either the loss of circulatory blood volume, as occurring during hemorrhage and hemodialysis, or the gain of circulatory blood volume, as occurring during transfusion, the arterial baroreceptors discharge leads to reflex sympathetic excitation and parasympathetic inhibition. This regulatory reflex causes the vasoconstriction of capacitance and resistance vessels and the rise of heart inotropic activity, thereby compensating for arterial and venous pressure reduction [8].

Hemodialysis treatment is largely used to maintain the body fluid composition and volume in patients with severe renal failure. In particular, by way of blood ultrafiltration, the dialyzer removes excess of both solute, uremic toxins and body water accumulated during the interdialysis period. Mathematical models of arterial pressure response to blood volume withdrawal have been proposed [9] and they provide a useful framework to analyze the role of short-term pressure control mechanisms on the pressure response during hypovolemia [10].

Physiological mathematical models are traditionally presented through a series of differential and algebraic equations with the option of a block diagram representing the interconnections of various subsystems [11]. In recent years, various specialized and general-purpose modelling software applications have been applied to the modelling and control of physiological systems, and are commonly divided into structure oriented and equation-oriented. Most software follows a causal modelling approach and requires either the explicit coding of mathematical model equations or the representation of systems in a graphical notation such as a block diagram, as occurs in SIMULINK [12], thus differing substantially from the more common representation of physiological knowledge. 
The object-oriented approach can offer many advantages in physiological system modelling and control when dynamics are given by a set of differential algebraic equations (DAE). This object-oriented approach can be implemented using modelling language such as MODELICA [13], or SIMSCAPE [14] among others. Both integrated environments are modeling languages that are ideally suited as architectural description languages for complex systems, which allow the system, subsystem, or component levels of a whole physical system to be described in increasing detail. In this way, individual components of the system model and its interconnections describe the underlying dynamic equations by allowing the design of the model to start from the inherent structure of the physical system [15]. Even though MODELICA is a powerful object-oriented simulation environment, the use of SIMSCAPE enables additionally the use of MATLAB Toolboxes, being this the main reason by which SIMSCAPE has been chosen as the modeling tool.

In this paper we describe the use of the SIMSCAPE modelling language under the MATLAB programming environment for the object-oriented modelling of the shortterm arterial pressure regulatory system. For this task we have followed an acausal hierarchical structure to represent the cardiovascular dynamic as a multi-compartmental system constituted by the arterial and venous tree together with the heart pump and the baroreceptor neural control system for a patient undergoing different situations as hemorrhage, blood transfusion or hemodialysis. Results obtained during simulation provide a physiological interpretative key to the patient's hemodynamic behavior during steady state and transient arterial pressure consequently induced.

\section{MODELING OF THE CARDIOVASCULAR CONTROL SYSTEM}

As a first approach, we can consider the cardiovascular system as a closed loop circuit constituted by the systemic and the pulmonary circulation in series with the heart pump configured by two ventricles with two valves each. The baroreceptor control system regulates the system peripheral resistance, venous elastances and heart rate (Fig. 1).

The cardiovascular model used here is based on previous studies by [16], implemented as an electric analog circuit and described formerly in [17]. The use of the analogy will enable us to define the voltage, current, charge, resistance, and capacitance in the electronic circuit as equivalent to the blood pressure, flow, volume, resistance, and compliance in the cardiovascular system.

According to this description, it can be related every element of the analog circuit with its analogous anatomic property of the circulatory system. On one hand, each ventricle will be described as an isovolumetric timedependent pressure generator in series with a time-varying elastance and a resistance. The unidirectional character of the inlet and outlet heart valves is modeled by an ideal diode in series with an electrical resistance. The atrial elastic characteristics are included in the venous compliances, which are modeled as capacitances in the circuit. On the other hand, the systemic and pulmonary circulations will be simply described by an RLC two-stage network, where resistances and inductances represent inertial and viscous properties of the blood flow, while capacitances correspond to the elastic properties of the vessel walls.

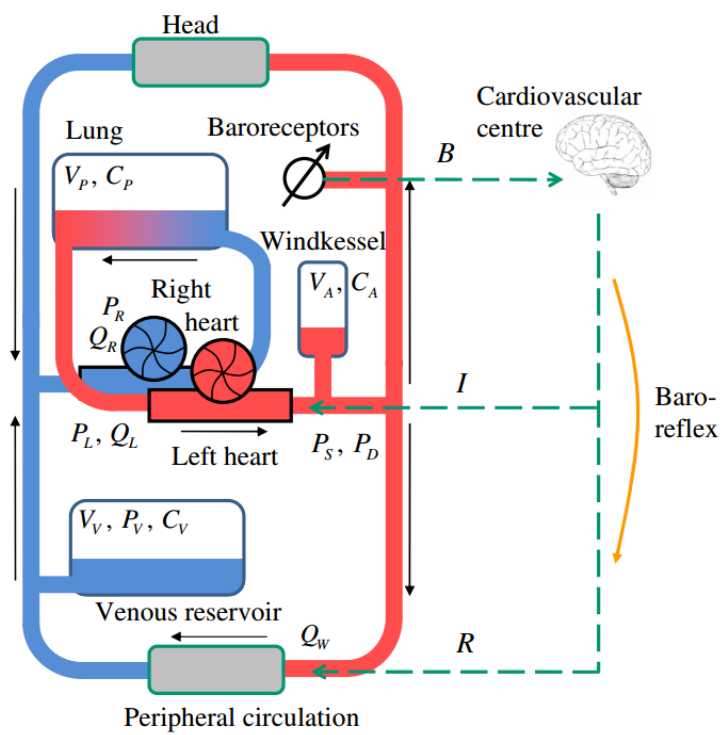

Figure 1. Schematic of the baroreceptor short-term pressure control system and whole cardiovascular circuit

The baroreceptor control takes effect on the unstressed venous volume, peripheral systemic resistance, ventricular elastances and heart rate according to the first-order transfer function expression with dead zone as reported in [8], where mean aortic pressure is taken as the common input (Fig. 2).

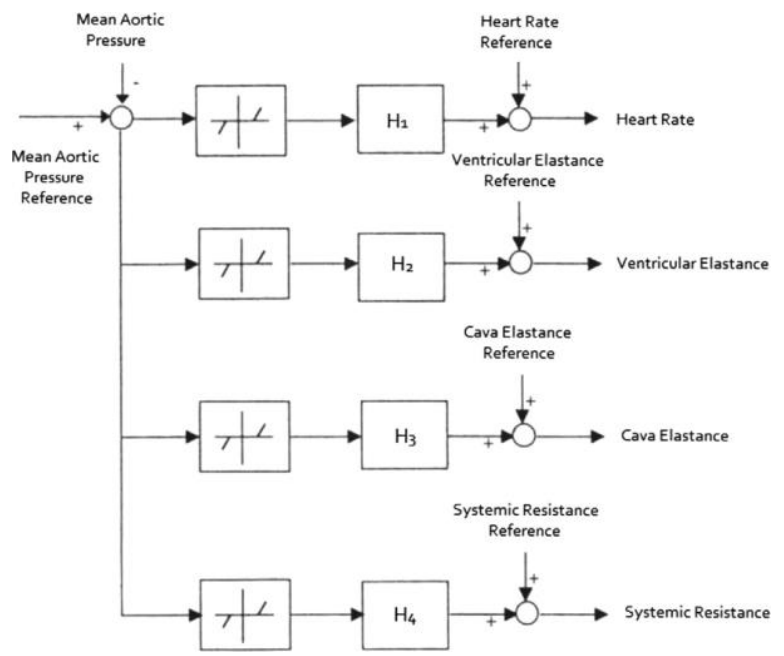

Figure 2. Schematic modeling of the baroreceptor regulating system 


\section{THE CARDIOVASCULAR CONTROL SYSTEM IN SIMSCAPE}

SIMSCAPE is an object-oriented language for modelling continuous complex physical systems for the purpose of computer simulation, and is primarily based on physical modeling rather than mathematical modeling. It also has multi-domain modelling capability, meaning that model components corresponding to physical objects from several different domains can be described and connected [18].

In SIMSCAPE, a model is defined as a collection of physical blocks which must be appropriately connected to define the dynamic system. The specific connection diagram together with the conservation laws applied determines the system dynamic equations.
Each of the constitutive components of the cardiovascular system defined in Fig. 1 was coded in SIMSCAPE starting from the set of equations defined in [16] by gathering the necessary blocks from the SIMSCAPE general purpose libraries together with those previously defined by the user. The baroreceptor control has also been modelled in SIMSCAPE by considering its effect through the variation on the parameters unstressed systemic venous volume, systemic resistance, ventricular elastances and heart rate.

Fig. 3 shows the complete cardiovascular circuit under baroreceptor control in SIMSCAPE also including the hemorrhage, transfusion and dialysis common block.

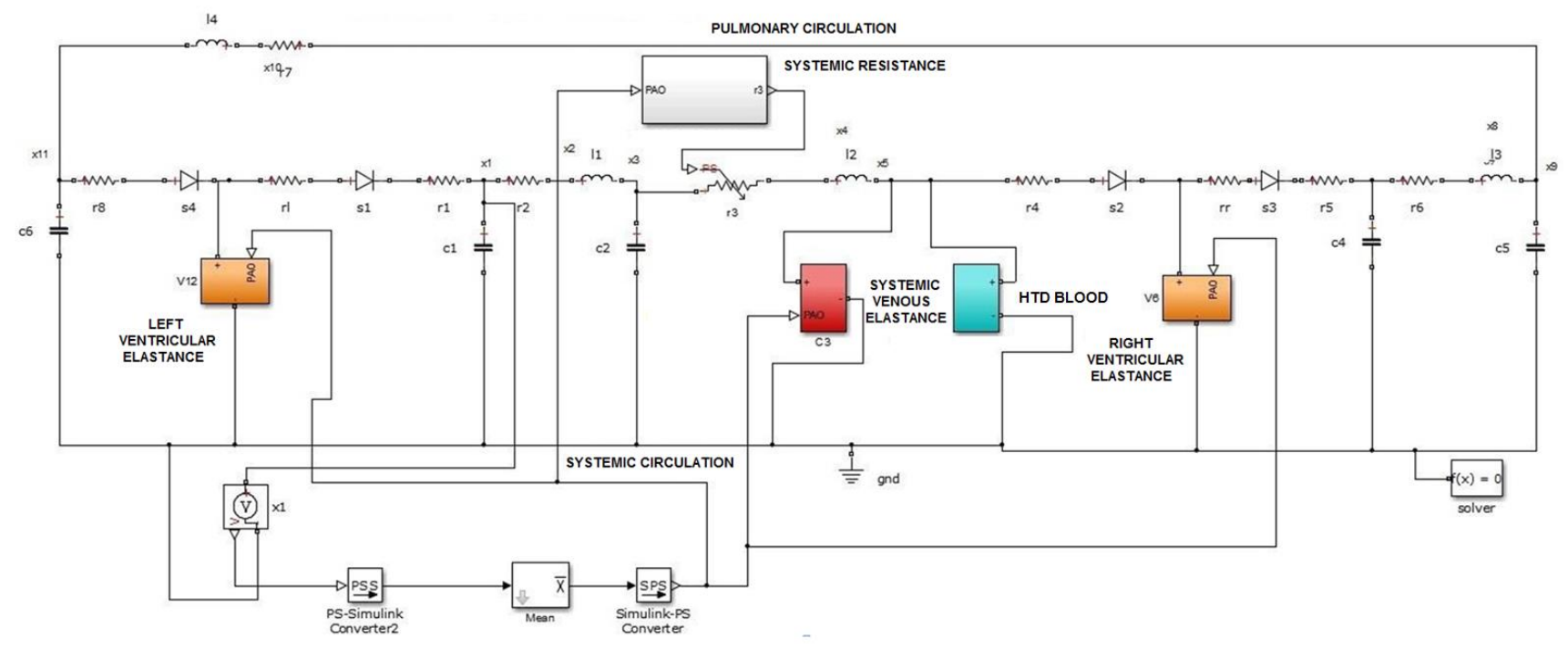

Figure 3. Schematic of the baroreceptor short-term pressure control system and whole cardiovascular circuit

The ventricles are modeled as controlled voltage sources. Each ventricle is composed of elastance and ventricular isovolumetric pressure generator time dependent, this time dependence is given by the activation function $a(t)$. The baroreceptor control mechanism influences both ventricular elastances through the mean aortic pressure taken as input (Fig. 4).
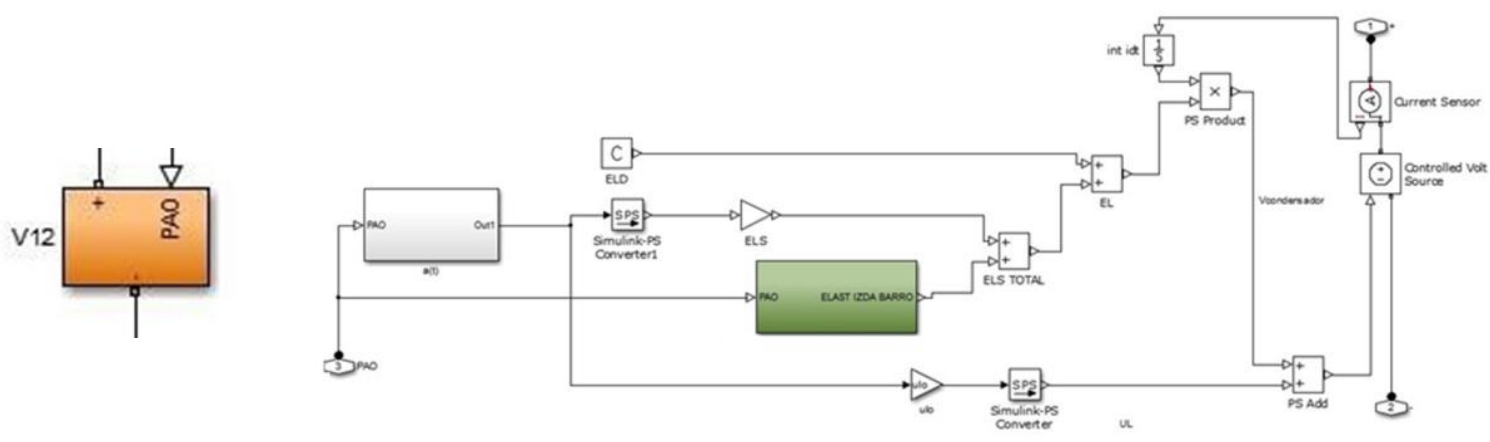

Figure 4. SIMSCAPE modeling of the ventricles under short-term baroreceptor control 
The systemic venous elastance (cava vein) is controlled by short-term control and modelled as a variable capacitor whose value os dependent on the first-order transfer function $H 3$ with dead zone (Fig. 5), and in the same way it is modelled the variable system resistance this time with a variable resistor and a different transfer function $H 4$.
In order to characterize the hemorrhage, transfusion and dialysis, a SIMSCAPE common block (HTD blood) has been built so as to allow the input and output of net blood flow. This module consists of a source of controlled intensity and a signal generator (Fig. 6).

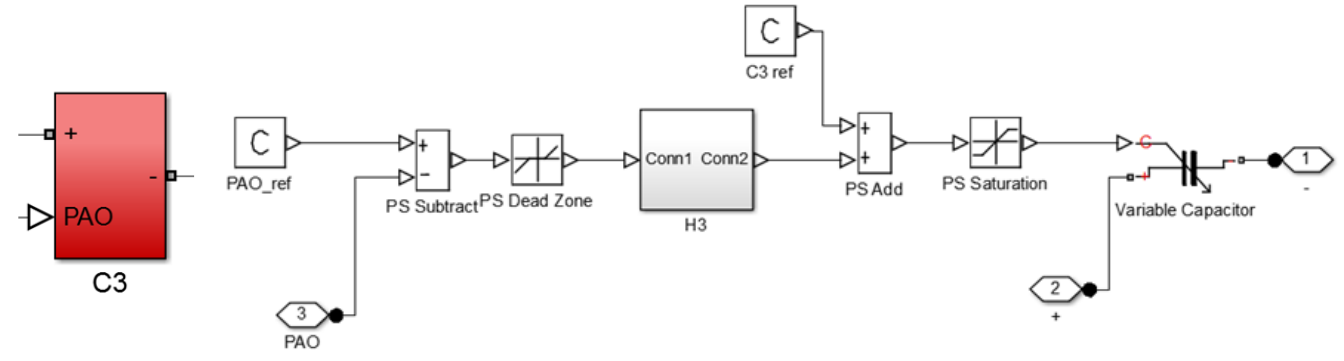

Figure 5. SIMSCAPE modeling of the venous systemic celastance under short-term baroreceptor control
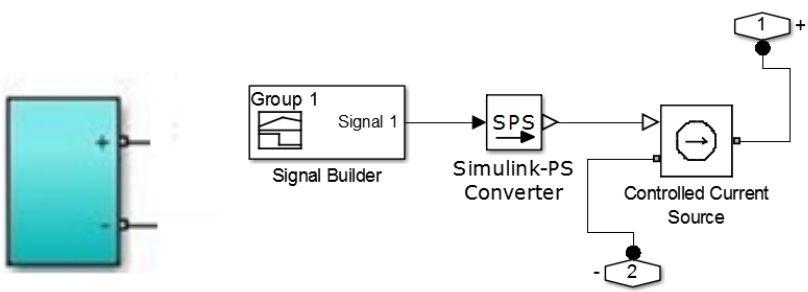

Figure 6. SIMSCAPE modeling of the hemorrhage, transfusion and dialysis common block

\section{SIMULATION RESULTS}

In order to test the performance of the short-term pressure control system several experiences were accomplished, both under physiological conditions by varying the reference values of resistance, elastance or heart rate in the baroreceptor control module and also by hemorrhage, liquid intake or blood transfusion and dialysis as external flow rates entering the circulatory system to evaluate the baroreceptor control. The results obtained took into account the validation tests performed previously with physiological data by [16].

In Fig. 7 it is shown the cardiovascular system response to a severe hemorrhage in terms of arterial pressure with and without short-term control. The baroreceptor tends to compensate for the decreasing in the arterial pressure.

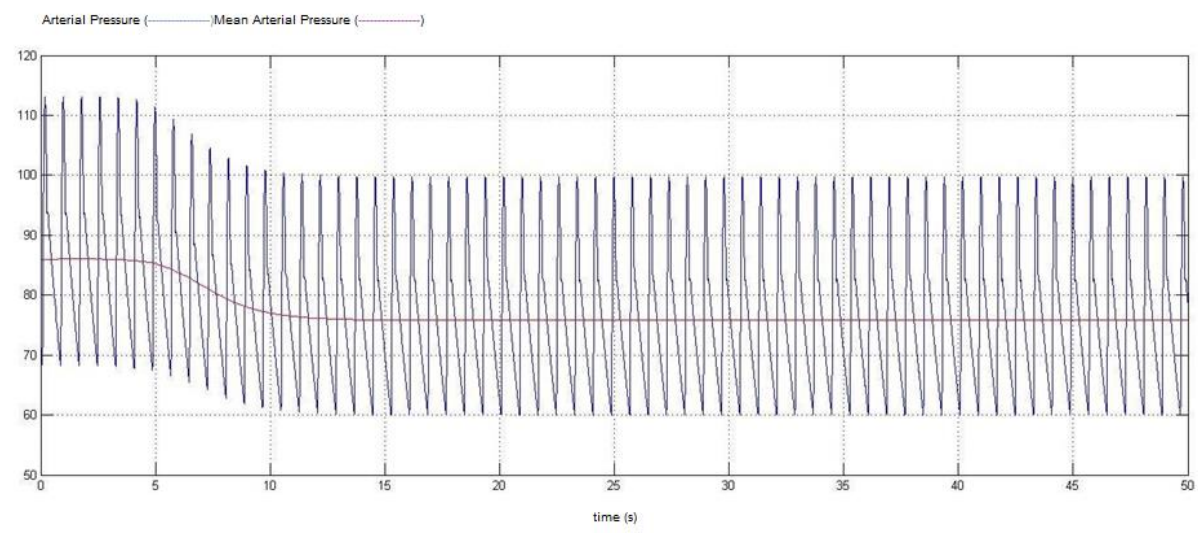




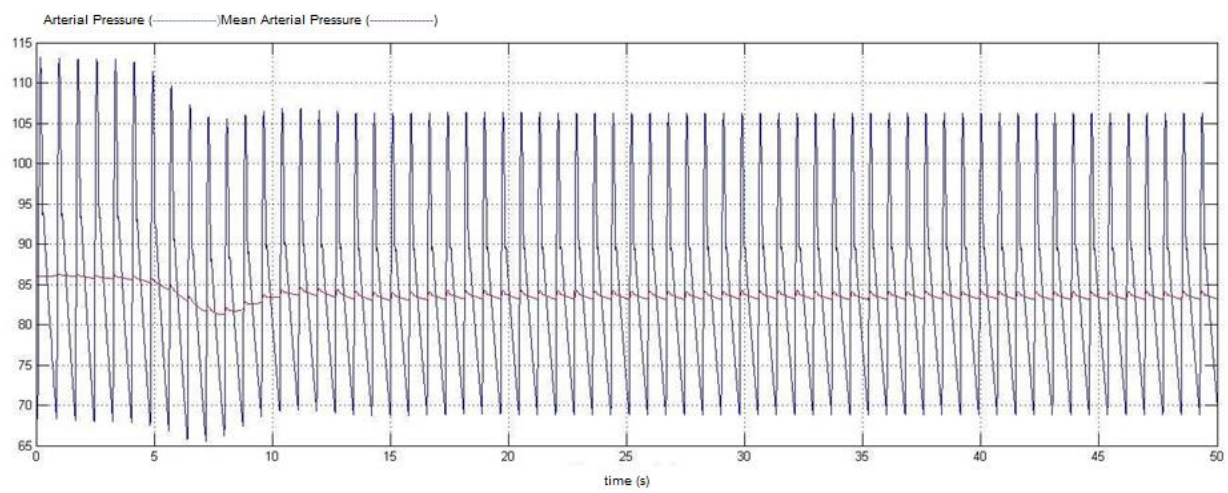

(b)

Figure 7. Cardiovascular system response response to a severe hemorrhage of $3000 \mathrm{ml} / \mathrm{min}$ during 3 sec without (a) and with (b) the baroreceptor compensation

Additionally, it was simulated a dialysis experience. Between dialysis sessions, the patient accumulates volume in the circulatory system due to fluid intake, resulting in increased blood pressure, and during the dialysis session that accumulated liquid is reduced.

In this this experience, the dialysis process was modelled with a combined liquid intake (transfusion)-hemorrhage pulse with and without baroreceptor control. As it was expected, the arterial pressure rises before the dialysis session since the patient ingests liquid and the blood removal by dialysis causes an abrupt pressure drop in a short period, provoking consequently an episode of acute hypotension. As one can see, the baroreceptors attenuate blood pressure changes over a wide range (Fig. 8) by reducing(increasing) systemic resistance and decreasing(increasing) venous elastance along with a proper decreasing(increasing) of heart rate (Fig. 9) and reduction(raise) of left and right ventricular elastances so as to restore the arterial pressure level to normal values before(after) the dialysis operation.

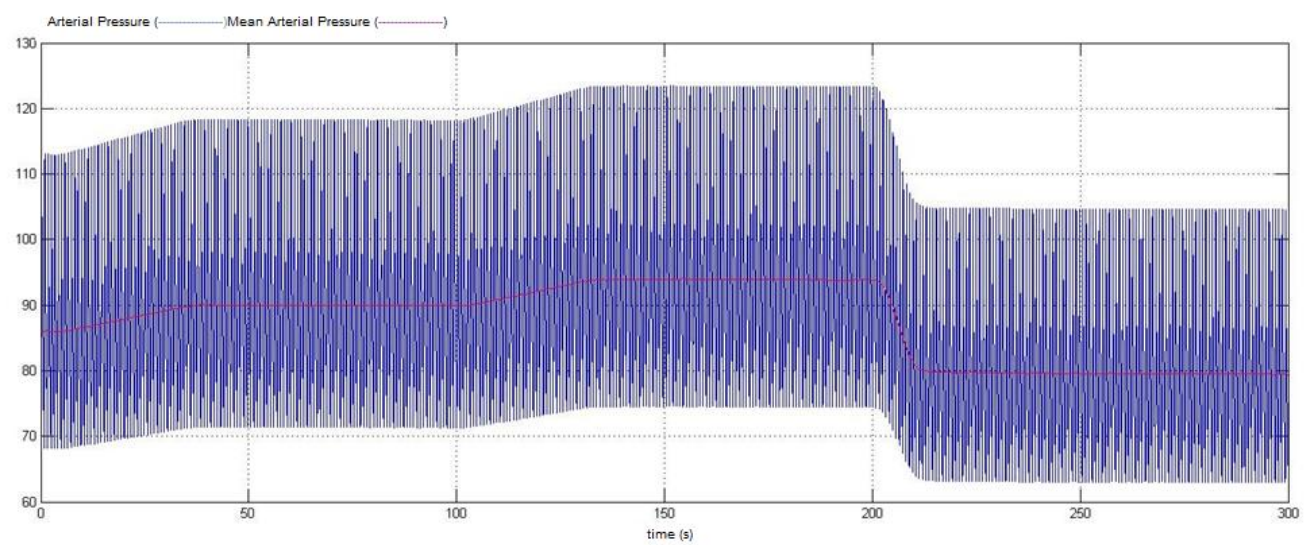




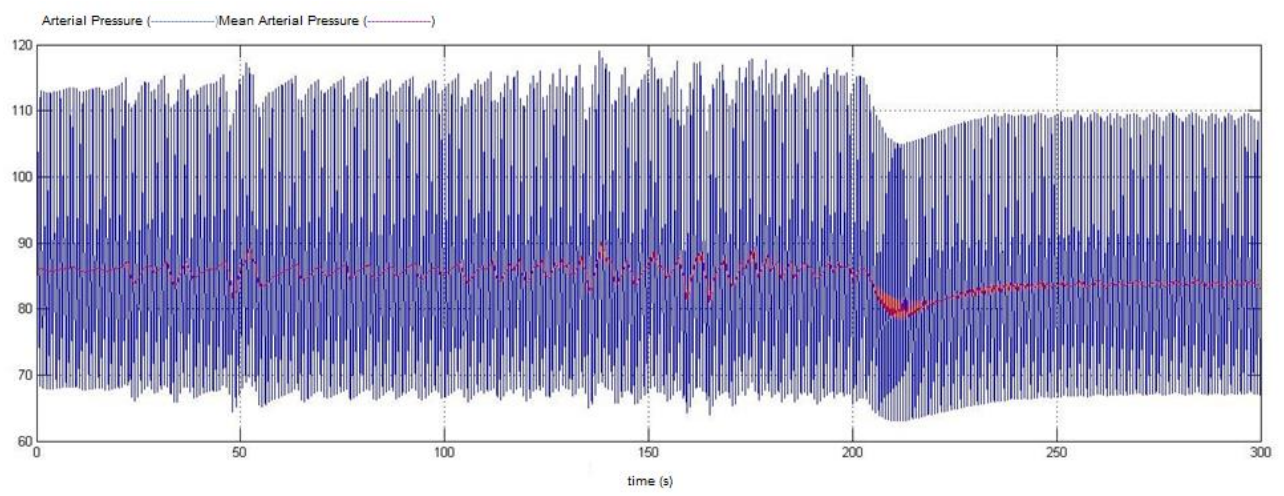

(b)

Figure 8. Cardiovascular system response response to liquid intake-dialysis experience of two transfusions of $120 \mathrm{ml} / \mathrm{min}$ for $30 \mathrm{sec}$ each and extraction of $1800 \mathrm{ml} / \mathrm{min}$ for $7 \mathrm{sec}$ without (a) and with (b) the baroreceptor compensation

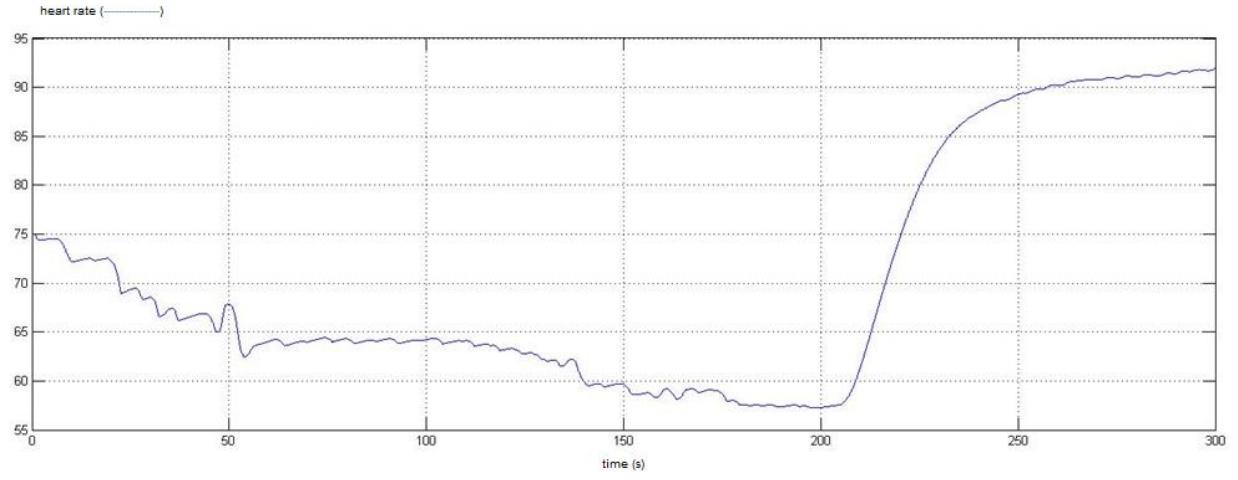

Figure 9. Baroreceptor control response in terms of heart rate to liquid intake-dialysis experience of two transfusions of $120 \mathrm{ml} / \mathrm{min}$ for $30 \mathrm{sec}$ each and extraction of $1800 \mathrm{ml} / \mathrm{min}$ for $7 \mathrm{sec}$

\section{V.CONCLUSION}

This paper presents an object-oriented model of the shortand pressure control of the cardiovascular system. An electric analog model of the cardiovascular system together with the baroreceptors mechanism was developed in SIMSCAPE using a hierarchical modelling strategy. The use of the SIMSCAPE approach considerably reduced the modeling effort and facilitated model reuse.

In this way, the use of SIMSCAPE facilitates model implementation as compared to the explicit coding of mathematical model equations or the representation of systems in a graphical notation as SIMULINK, so it can be regarded as a suitable tool to simulate physiological and pathological scenarios as it occurs in dialysis.

Future work will consider a more detailed module to describe the dialysis dynamics in terms of liquid and solutes transfer so as to evaluate alternate interdialysis treatment periods and dialysate liquid composition.

\section{REFERENCES}

[1] A.C. Guyton, J.E. Hall, Textbook of Medical Physiology, ninth ed., W.B. Saunders, New York, NY, 1996.

[2] R.M. Berne, M.N. Levy, Cardiovascular Physiology, Mosby, St. Louis, 1997.

[3] C.F. Rothe, J.M. Gersting, "Cardiovascular interactions: an interactive tutorial and mathematical model", Am. J. Physiol. Adv. Physiol. Educ, vol. 26, pp. 98-109, 2002.

[4] J.J. Batzel, F. Kappel, D. Schneditz, H.T. Tran, "Cardiovascular and Respiratory Systems: Modeling, Analysis, and Control “, in: Frontiers in Applied Mathematics, SIAM, 2006.

[5] G.M. Raymond, E. Butterworth, J.B. Bassingthwaighte, "JSIM: free software package for teaching physiological modeling and research", Exp. Biol, vol. 280, pp. 102-107, 20003.

[6] S.R. Abram, B.L. Hodnett, R.L. Summers, T.G. Coleman, R.L. Hester, "Quantitative circulatory physiology. An integrative mathematical model of human mathematical model of human physiology for medical education", Adv. Physiol. Educ., vol. 31, pp. 202-210, 2007.

[7] V.I. McLoone, J.V. Ringwood, B.N. VanVliet, "Graphical simulation environments for modeling and simulation of integrative physiology", Comput. Meth. Program. Biomed, vol.102 (3), pp. 295-304, 2011.

[8] Y. Mitamura, "Control aspects of the circulatory system", in: IFAC Control Aspects of Biomedical Engineering, pp. 37-51, 1987. 
[9] M. Ursino, L. Coli, E. Magosso, P. Capriotti, A. Fiorenzi, P. Baroni S. Stefoni, "A mathematical model for the prediction of solute kinetics, osmolarity and fluid volume changes during hemodiafiltration with on-line regeneration of ultrafiltrate (HFR)", Int. J. Artif. Organs, vol. 29, pp. 1031-1041, 2006.

[10] S. Cavalcanti, S. Cavani, A. Ciandrini, G. Avanzolini, "Mathematical modeling of arterial pressure response to hemodialysis-induced hypovolemia “,Com. Bio. Med., vol. 36, pp. 128-144, 2006.

[11] J. Kofranek, J. Rusz, "Restoration of Guyton's diagram for regulation of the circulation as a basis for quantitative physiological model development", Physiol. Res, vol. 59, pp. 897-908, 2010.

[12] S.T. Harris, Introduction to Simulink: With Engineering Applications, Orchard Publications, 2008.

[13] P. Fritzson, Introduction to Modeling and Simulation of Technical and Physical Systems with Modelica, Wiley-IEEE Press, 2011.

[14] http://www.mathworks.com/help/physmod/simscape/, 2013.

[15] G. Zauner, D. Leitner, F. Breitenecker, "Modeling structural dynamic systems in Modelica/Dymola, Modelica/Mosilab and AnyLogic", in: Proceedings of the International Workshop on Equation-Based Oriented Languages and Tools, 2007.

[16] G. Avanzolini, P. Barbini, A. Cappello, G. Cevenini, “ CACDS simulation of the closed-loop cardiovascular system", Int. J. Biomed. Comput., vol. 22 , pp. 39-49, 1988.

[17] J. Fernandez de Canete, P. Del Saz-Orozco, D. Moreno-Boza, E. Duran-Venegas, "Object-oriented modeling and simulation of the closed loop cardiovascular system by using SIMSCAPE." Comp. Bio. Med, vol. 43(4), pp. 323-333,2013

[18] T.J. Hassell, W. Weaver, A.M. Oliveira, "Using Matlab's Simscape modeling environment as a simulation tool in power electronics and electrical machines courses", in: Frontiers in Education Conference 2013 IEEE, pp. 477-483, 2013. 